\title{
Scene Data Fusion: Real-Time Standoff Volumetric Gamma-Ray Imaging
}

\author{
Ross Barnowski ${ }^{\mathrm{a}}$, Andrew Haefner ${ }^{\mathrm{b}}$, Lucian Mihailescu ${ }^{\mathrm{b}}$, Kai Vetter ${ }^{\mathrm{a}, \mathrm{b}}$ \\ ${ }^{a}$ Department of Nuclear Engineering, UC Berkeley, 4155 Etcheverry Hall, MC 1730, \\ Berkeley CA 94720, United States of America \\ ${ }^{b}$ Lawrence Berkeley National Lab - Applied Nuclear Physics, 1 Cyclotron Road, Berkeley \\ CA, 94720, United States of America
}

\begin{abstract}
An approach to gamma-ray imaging has been developed that enables near realtime volumetric (3D) imaging of unknown environments thus improving the utility of gamma-ray imaging for source-search and radiation mapping applications. The approach, herein dubbed scene data fusion (SDF), is based on integrating mobile radiation imagers with real-time tracking and scene reconstruction algorithms to enable a mobile mode of operation and 3D localization of gamma-ray sources. A 3D model of the scene, provided in real-time by a simultaneous localization and mapping (SLAM) algorithm, is incorporated into the image reconstruction reducing the reconstruction time and improving imaging performance. The SDF concept is demonstrated in this work with a Microsoft Kinect RGB-D sensor, a real-time SLAM solver, and a cart-based Compton imaging platform comprised of two 3D position-sensitive high purity germanium (HPGe) detectors. An iterative algorithm based on Compton kinematics is used to reconstruct the gamma-ray source distribution in all three spatial dimensions. SDF advances the real-world applicability of gamma-ray imaging for many search, mapping, and verification scenarios by improving the tractiblity of the gamma-ray image reconstruction and providing context for the $3 \mathrm{D}$ localization of gamma-ray sources within the environment in real-time.
\end{abstract}

Keywords: Compton imaging, Gamma-ray imaging, SLAM, 3D imaging, Volumetric imaging, Data fusion

Preprint submitted to Nuclear Instruments and Methods in Physics Research AAugust 7, 2015 


\section{Introduction}

2 Gamma-ray imaging has many applications in a wide variety of fields, including gamma-ray astronomy, medical imaging, and nuclear security and safe4 guards. Additionally, the application of gamma-ray imaging to environmental 5 monitoring and nuclear contamination remediation scenarios is being actively researched $[1,2,3]$. There exist several directional imaging methods that characterize the spatial distribution of gamma-ray sources in 2D. Compton and coded-aperture based methods have been used to provide sensitivity to the spatial distributions of cosmic gamma-ray sources[4, 5], while collimator-based approaches have long been a staple in diagnostic imaging [6]. These methods have also been adopted for nuclear safeguards and security applications such as nuclear facility monitoring, source-search, and emergency response, as well as applications in nuclear contamination remediation. Many of these applications would further benefit from volumetric imaging; i.e. the ability to characterize gamma-ray source distributions in all three spatial dimensions, including depth. Previous work has investigated combining conventional 2D gamma-ray projection imaging techniques with 3D models of the scene[7]. This work demonstrates the value of the context provided by merging with 3D models, but falls short of data fusion as defined here because the 3D model information is not incorporated into the gamma-ray imaging technique. Reference [8] extends this work to true volumetric imaging via data fusion with the same data. Volumetric imaging is achieved in biomedical applications by leveraging the self-contained, near-field nature of the imaging domain. In some cases, this allows full or partial tomographic methods [9] to be applied for both transmission [10] and emission $[11,12]$ based imaging modalities. There are other approaches that don't use tomographic reconstruction, but nevertheless rely on the self-contained nature of the imaging domain $[13,14,15]$. There are challenges unique to nuclear security and safeguards applications that preclude the direct application of these 3D methods: large variability of the size and complexity of the imaging environment; the inability to acquire orthogonal projections; and comparatively 
low count rates in real-world scenarios. Scene data fusion overcomes these challenges for standoff imaging, where "standoff" here represents distances in the range of one to tens of meters commensurate with the scale relevant for many nuclear security and safeguards applications.

The SDF approach is based on previous work[8] in which $3 \mathrm{D}$ models of the scene were incorporated into a volumetric gamma-ray image reconstruction algorithm. In that work, the detector had to be manually positioned and the models were laboriously constructed by hand using measurements taken with a laser range finder. Furthermore, thousands of Compton cones were used in the image reconstruction. The SDF approach surmounts these limitations and enables a mobile mode of operation for portable radiation imaging devices. This is achieved by integrating a mobile radiation imaging platform with a realtime tracking method that provides an estimate of the location and orientation (i.e. pose) of the system as it moves throughout the scene. By synchronizing and spatially registering the pose estimates with the data from the radiation imager, a track throughout the scene populated with gamma-ray interactions is produced, serving as the input to a volumetric image reconstruction algorithm. In practice, these data alone often result in prohibitively noisy images due to the real-world imaging challenges detailed above. The 3D model of the scene can then be used to spatially constrain the gamma-ray image reconstruction, improving image quality in terms of localization accuracy and reduced image noise. SDF is defined by the utilization of both the localization and mapping capability provided by directly integrating SLAM-solvers and auxiliary sensors with radiation imaging systems, enabling a mobile imaging mode capable of $3 \mathrm{D}$ gamma-ray source localization. In principle, there are many ways by which the complementary data can be utilized in the reconstruction due to the multitude of imaging modalities (collimated, Compton) and the various approaches to the inversion (e.g. filtered back projection, iterative methods). This work focuses on a specific approach based on Compton imaging, which is particularly suited for source search and gamma-ray mapping applications due to the inherent wide field of view and sensitivity over a large range in gamma-ray energies[16]. 


\section{System Implementation - Volumetric Compton Imager}

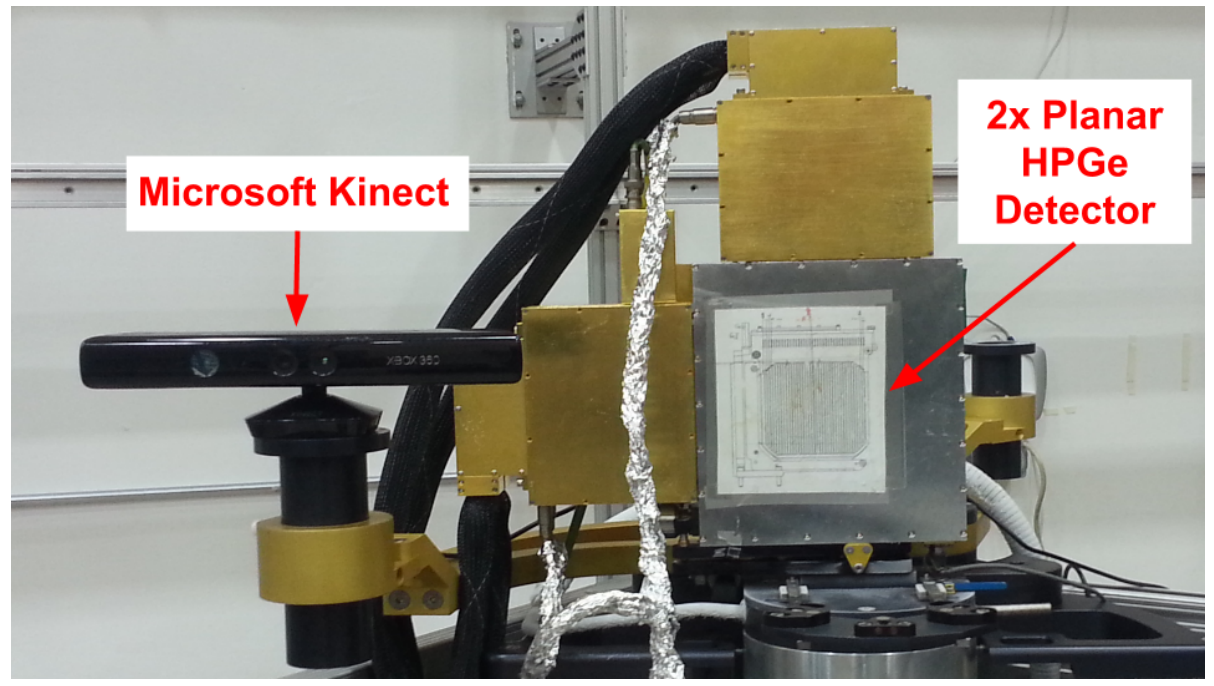

Figure 1: The VCI system. The Kinect is mounted near the detector cryostat and kept stationary with respect to the detector. During volumetric measurements, the entire cart is pushed through the scene thus the detector and Kinect move together.

The gamma-ray event reconstruction and tracking used in this work is not optimized for sensitivity as the focus of this work is real-time imaging. As a result, the position resolution and Compton imaging efficiency are limited, imposing an upper bound on the detection rate for imagable Compton events. Real-time imaging is demonstrated in section 4 despite the artificially limited

\footnotetext{
${ }^{1}$ In practice, issues with discrete electronics for several channels cause a slight reduction of the sensitive volume.
} 
Compton event rate, illustrating the efficacy of SDF even for relatively inefficient gamma-ray imaging systems.

The SLAM-solver chosen for this work is known as RGBDSlam, and is presented in full detail in [17]. The system utilizes a Microsoft Kinect sensor that provides both RGB images and dense 3D point cloud representations of the environment at a frame rate $\geq 15 H z$ and an effective range of $4-6 m$ depending on the configuration. The algorithm publishes estimates of the pose of the RGB-D sensor as well as an aggregate point cloud model of the scene in real time. The coordinate frame of the scene model is set by the first frame acquired from the Kinect, thus subsequent pose estimates represent a transformation from the current Kinect location to the scene coordinate frame. The RGB-D sensor is kept rigid with respect to the detectors on the VCI cart, and the relative transformation between the coordinate frame of the detector and the Kinect is determined. This registration transform is then applied to the Compton events collected by the detector to correctly orient them in the scene coordinate frame. By synchronizing the tracked pose of the detector with the Compton events, a full Compton event history along the track of the detector is compiled and updated in real time. The radiation image as well as the imaging space are continuously recomputed as new Compton events are measured along the detector track.

\section{Volumetric Image Reconstruction Algorithm}

Given the ability to sample gamma-ray distributions with a mobile Compton imager, the challenge of determining the 3D spatial distribution of gamma-ray sources remains. Current far-field reconstruction methods developed for astronomy and nuclear security [18] based on filtered back-projection are not directly applicable to the $3 \mathrm{D}$ problem due to the higher dimensionality of the imaging space. Perhaps the greatest challenge is the arbitrary nature of the imaging environment itself; in general, it is unlikely that the imager will be able to obtain the orthogonal projections necessary for a full tomographic reconstruction. 
The reconstruction algorithm must also be able to handle the low count rates expected for real-world imaging applications. These considerations led to the development of an ML-EM reconstruction algorithm for far-field 3D Compton imaging with additional modifications to incorporate the complementary scene data.

\subsection{ML-EM Overview}

A Poisson likelihood model is used to relate the gamma-ray source distribution to the data measured along the detector path. In the case of Compton imaging, the measured Compton cones describe from which voxels in the imaging space the gamma-rays could have originated. The EM portion of the algorithm maximizes the Poisson likelihood given this description of the imaging space [19]. List-mode reconstruction is used in this case, thus the sensitivities are not computed from the system matrix; uniform sensitivities are used instead. Furthermore, the system matrix is computed using a $3 \mathrm{D}$ cone with a Gaussian width, and voxels are assumed to be points in space. These simplifications facilitate real-time reconstruction, but are approximatioins that can result in imaging artefacts. However, it has been empirically determined that these simplifications are not prohibitive for localizing point sources in 3D.

\subsection{Scene Data Constraint}

As the 3D scene model is updated by RGBDSlam, the imaging space is recomputed based on the extent of the detector track within the model. The algorithm can use the scene model to compute an occupancy grid of the voxelized imaging space and limit the solution to the occupied voxels. This simple fusion technique is based on the assumption that gamma-ray sources are on the surface of or contained within objects and are not present in air or other void space. The image space can also be computed without restricting the solution for scenarios where the model is incomplete due to, for example, the limited FOV of the Kinect. Restricting the reconstruction to the 3D scene can be toggled on or off by the user as a more complete model becomes available. This ensures that the 


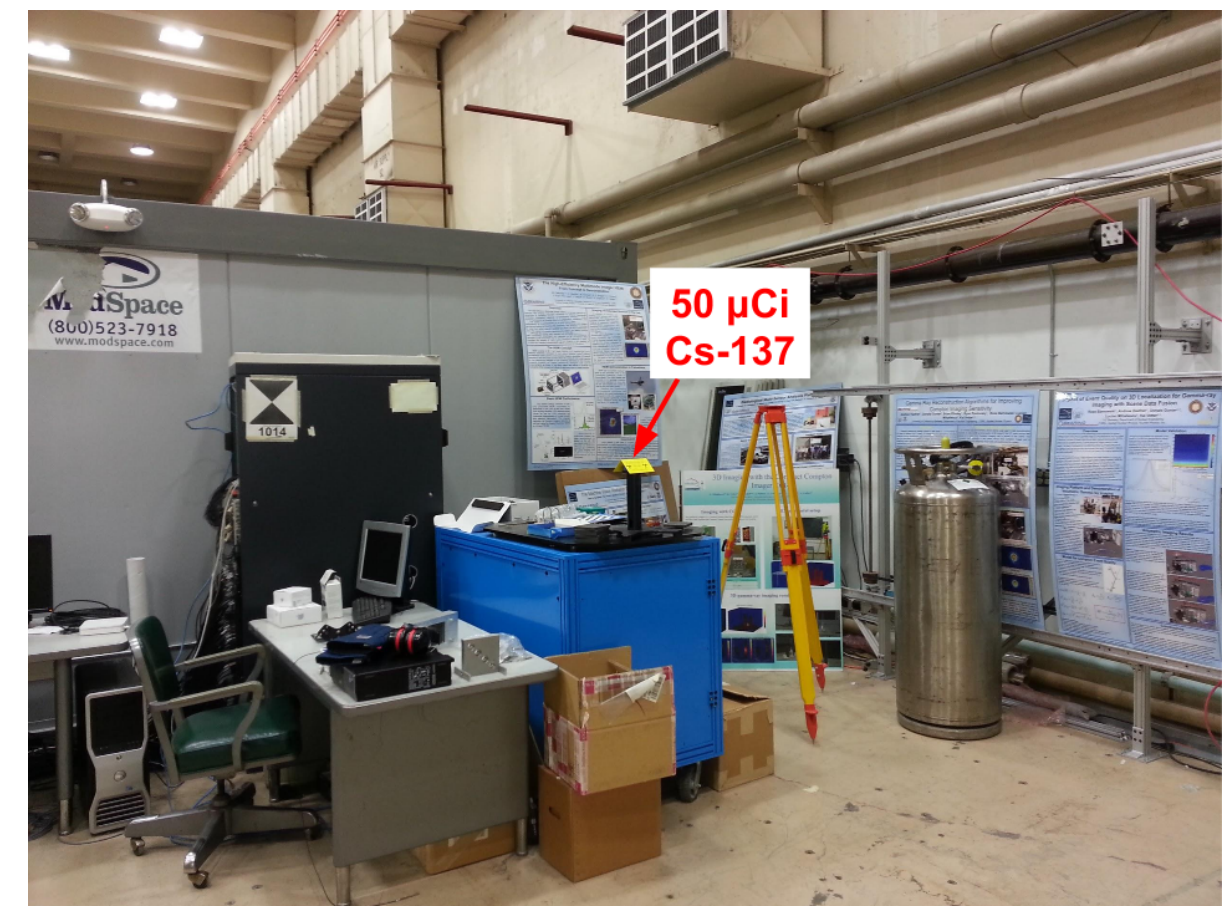

Figure 2: Environment in which the demonstration measurement was taken. The VCI moves from left to right on a slightly curved path.

algorithm is capable of running without a scene model, though the additional constraint on the imaging space is often necessary to produce accurate images of point sources.

\section{Results}

Presented here are the results of a single measurement to demonstrate the SDF approach applied to a gamma-ray source search and mapping application. A $50 \mu \mathrm{Ci}{ }^{137} \mathrm{Cs}$ point source is placed in the cluttered, indoor laboratory environment shown in figure 2. The Location of $50 \mu \mathrm{Ci}{ }^{137} \mathrm{Cs}$ point source is indicated by the red arrow in figure 2 .

The VCI is pushed along a slightly curved path facing the scene shown in figure 2. The total length of the cart path is $4.6 \mathrm{~m}$ with the source-detector distance reaching a minimum of about $1.5 \mathrm{~m}$ near the center of the track. It 
took 124 seconds to push the cart along this trajectory, collecting a total of 85 full-energy Compton events along the track.

The data collected during the run are passed separately to the volumetric ML-EM algorithm in both modes: the first without restricting the reconstruction to the $3 \mathrm{D}$ model, and the second with this constraint. In each case, the $3 \mathrm{D}$ model of the scene reconstructed via RGBDSlam is plotted in gray-scale (in order to provide contrast with the radiation image), while isosurfaces computed from the gamma-ray images are displayed in color. The red line represents the tracked position of the detector as it was pushed through the scene, while the white spheres represent points along that track where full-energy Compton events were recorded. Finally, the blue arrows emanating from the white spheres represent the scatter-axes of the Compton events. As with traditional Compton imaging, the scatter axis is defined by the location of the first two gamma-ray interactions (assuming the first interaction is Compton scattering), while the opening angle of the Compton cone is computed from kinematics given by the energy deposited in the first Compton scatter interaction and the energy of the incident photon. Since only the scattering direction is indicated by the arrows, it is possible to have arrows pointed at any orientation relative to the source location (arrows pointing away from the source correspond to Compton backscatter events). The imaging algorithm runs in near real-time: a non-optimized, implementation takes $200 \mathrm{~ms}$ to compute the back projection, and another 200 ms to complete $10 \mathrm{EM}$ iterations on a single core (no multithreading) of an intel if $4600 \mathrm{U}$ at $2.1 \mathrm{GHz}$. The number of iterations was chosen to balance the real-time requirement with image convergence, with ten being empirically determined to be sufficient for the reconstruction of point sources. The results presented here were computed offline so that the different imaging modes could be directly compared. 


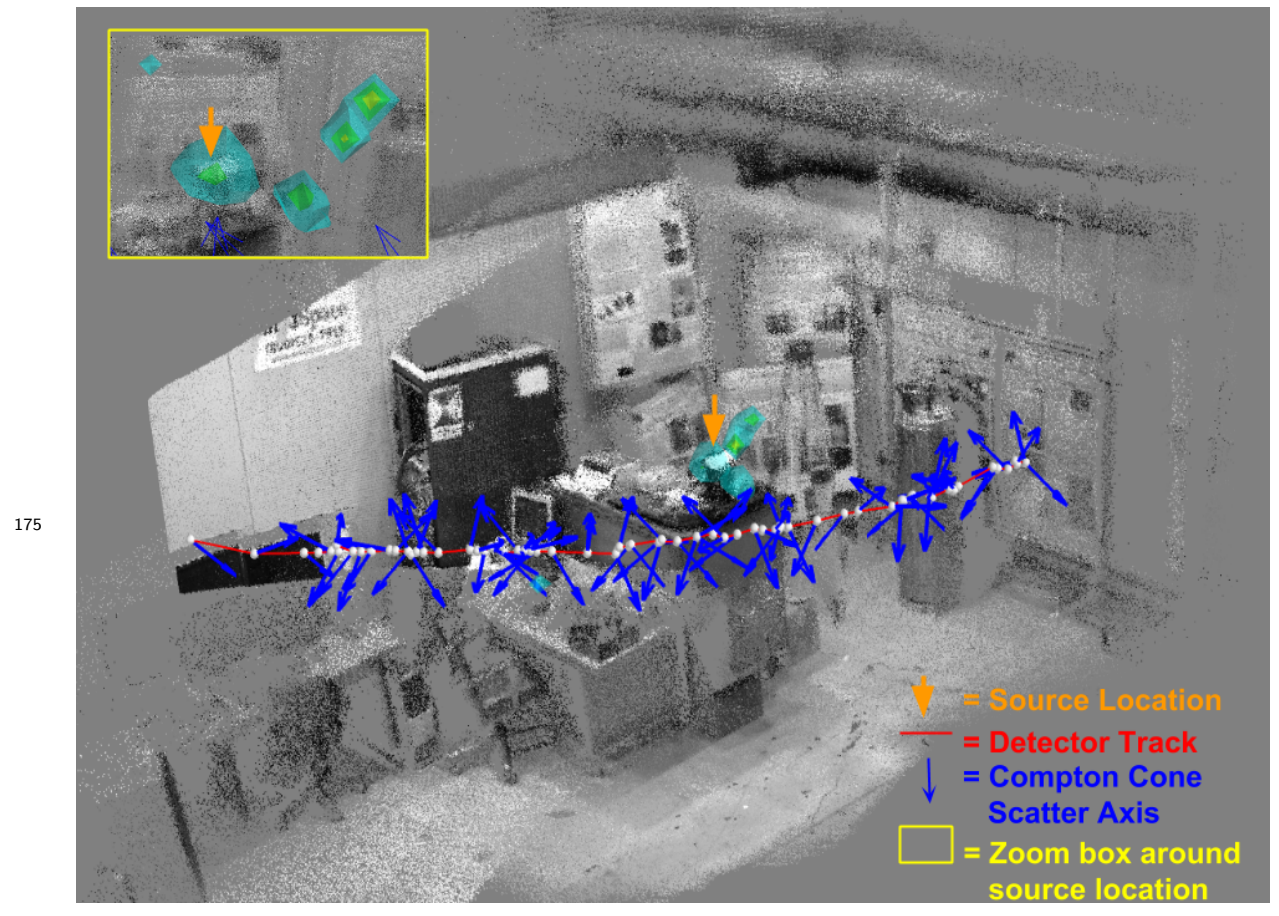

Figure 3: Volumetric gamma-ray image reconstructed without the point cloud constraint. The scene model is still shown for context, but was not used in the reconstruction.

Figure 3 contains the baseline image when the model is not used, i.e. the entire space along the detector path is voxelized and reconstructed without any occupancy constraint. The reconstruction of the source location is comparatively inaccurate and blurred in the direction perpendicular to the detector track. Image artefacts are also clearly visible in regions well away from the source location. In contrast, the artefacts are eliminated and the radiation image more accurately reflects the true source location when the model is used to restrict the imaging space, shown in figure 4 . The magnitude of the reduction in the imaging space using the scene model constraint will obviously depend on the scene being viewed. For this particular measurement, the original image space contained 1.65 million voxels with only 28,000 remained after applying the constraint, reducing the imaging space by nearly a factor of 60 . While results from only a single measurement are presented here, the SDF method is robust for a variety of point source measurements over a range of photon energies, 
detector paths, and source distances.

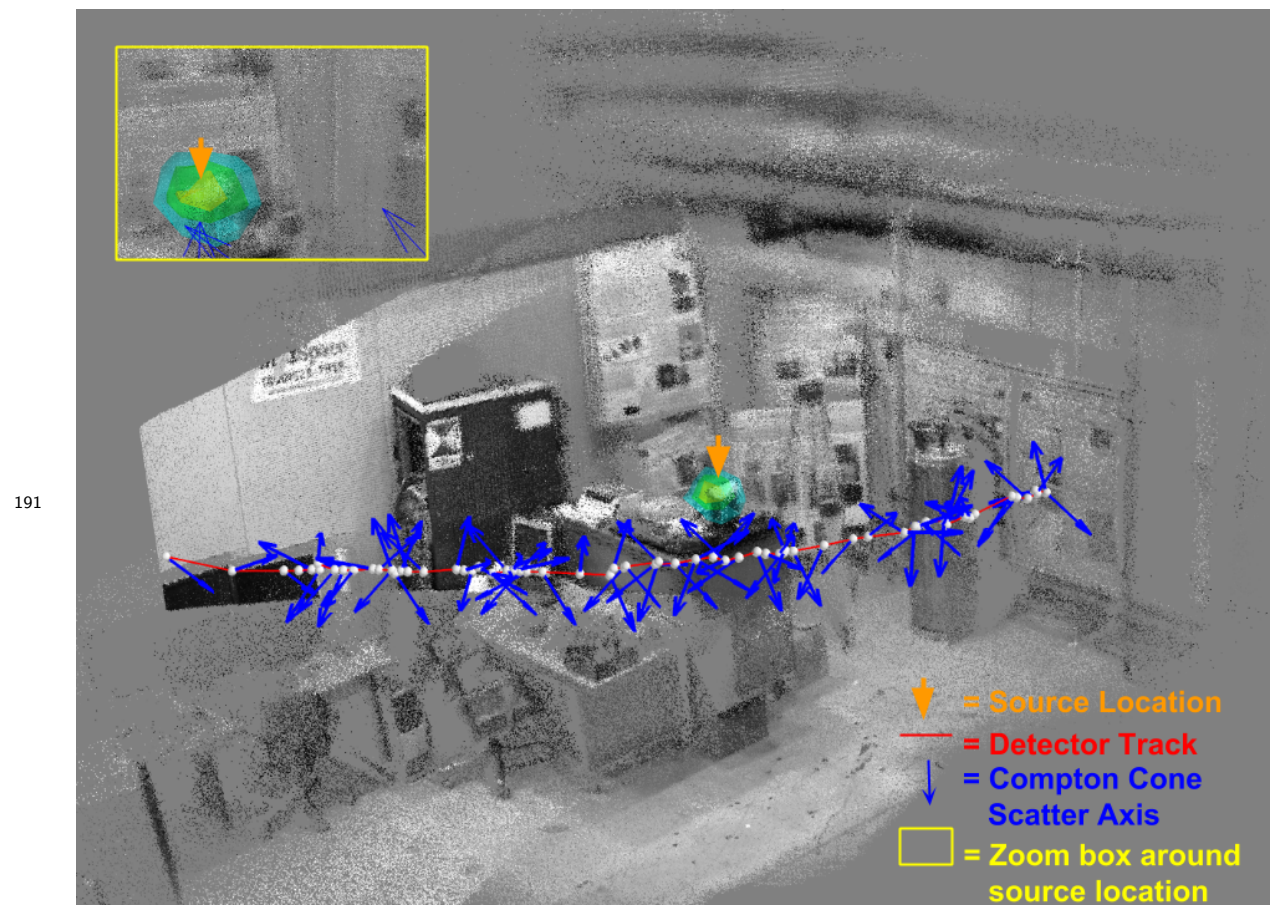

Figure 4: Volumetric gamma-ray image reconstructed with the point cloud constraint.

A second measurement demonstrates the efficacy of SDF for mapping more complicated gamma-ray source distributions. Two ${ }^{137} \mathrm{Cs}$ point sources, each with an activity of $20 \mu \mathrm{Ci}$ were placed about $30 \mathrm{~cm}$ apart. The VCI was pushed along an approximately linear path in front of the sources. Each source was about $1 \mathrm{~m}$ away from the detector at closest approach, and the measurement time was 6 minutes and 48 seconds. 315 full-energy Compton cones were collected. 


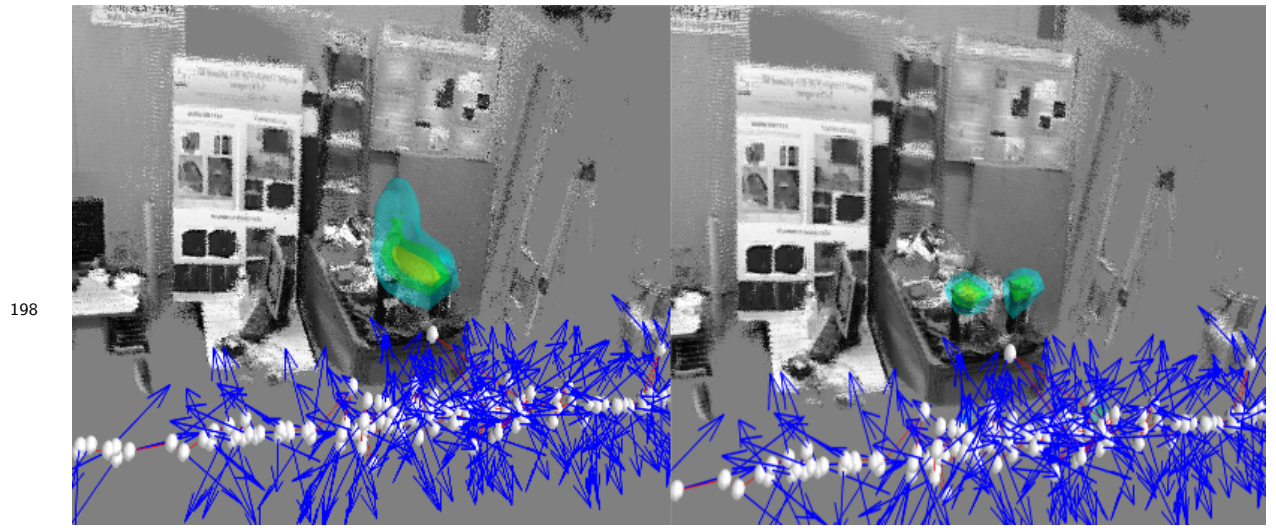

Figure 5: Left: Unconstrained. Right: With model constraint.

The unconstrained scenario results in a single hotspot blurred in the lateral direction, while the constrained case clearly distinguishes the sources. This result demonstrates the conceptual capability of the method to accurately reconstruct more complicated source distributions. It should be noted however that the ability to reconstruct distributed gamma-ray sources in general depends on proper weighting of geometric and temporal factors which are not explicitly accounted for in the present treatment.

\section{Conclusions}

Standoff volumetric gamma-ray imaging has many potential applications in nuclear security, safeguards, and other source search or gamma-ray source mapping scenarios. The additional information in the depth dimension over existing 2D imaging methods is valuable for many of these applications, and is a necessary first step towards the ability to quantify source activities from standoff measurements. While volumetric imaging has been a staple of medical diagnostics for decades, the additional challenges faced in standoff scenarios, particularly limited sampling capabilities; low count rates; and undefined, largescale imaging domains; necessitate the development of new methods. This work demonstrates that these challenges can be overcome by incorporating complementary data about the imaging environment into the gamma-ray image reconstruction. The real-time tracking capability of SLAM combined with mobile 
gamma ray sensors not only enables real-time volumetric gamma-ray imaging, but allows for the exploration of applications based on detector tracking without the need for highly-specialized instruments. There is much to be explored and evaluated in this regime, but it is clear that SDF provides new capabilities that can address issues that are fundamental limitations in static scenarios, such as $\frac{1}{r^{2}}$. The VCI system with which the presented results were obtained was not optimized for imaging efficiency or precision, yet accurate volumetric images are consistently reconstructed using fewer than 100 Compton events. The applicability of gamma-ray imaging to a wide variety of radiation search and mapping scenarios is greatly enhanced via SDF, providing 3D localization capability with environmental context in real time without requiring significant sensitivity improvements over modern portable gamma-ray imaging systems.

\section{Acknowledgements}

This material is based upon work supported by the Department of Energy National Nuclear Security Administration under Award number DE-NA0000979. Additionally, this material is based upon work supported by the U.S. Department of Homeland Security under the Grant Award Number contract ECCS1140069. This support does not constitute an express or implied endorsement on the part of the Government.

[1] T. Takahashi, S. Takeda, H. Tajima, S. Watanabe, Visualization of radioactive substances with a si/cdte compton camera, in: Nuclear Science Symposium and Medical Imaging Conference (NSS/MIC), 2012 IEEE, IEEE, 2012, pp. 4199-4204.

[2] J. Kataoka, A. Kishimoto, T. Nishiyama, T. Fujita, K. Takeuchi, T. Kato, T. Nakamori, S. Ohsuka, S. Nakamura, M. Hirayanagi, et al., Handy compton camera using $3 \mathrm{~d}$ position-sensitive scintillators coupled with large-area monolithic mppc arrays, Nuclear Instruments and Methods in Physics Research Section A: Accelerators, Spectrometers, Detectors and Associated Equipment 732 (2013) 403-407.

[3] D. Tomono, T. Tanimori, H. Kubo, A. Takada, T. Mizumoto, Y. Mizumura, T. Sawano, Y. Matsuoka, S. Komura, S. Nakamura, et al., First application to environmental gamma-ray imaging with an electron tracking compton 
camera, in: Nuclear Science Symposium and Medical Imaging Conference (NSS/MIC), 2013 IEEE, IEEE, 2013, pp. 1-5.

[4] S. Boggs, W. Coburn, D. Smith, J. Bowen, P. Jean, J. Kregenow, R. Lin, P. von Ballmoos, Overview of the nuclear compton telescope, New Astronomy Reviews 48 (1) (2004) 251-255.

[5] E. Caroli, J. Stephen, G. Di Cocco, L. Natalucci, A. Spizzichino, Coded aperture imaging in $\mathrm{x}$-and gamma-ray astronomy, Space Science Reviews 45 (3-4) (1987) 349-403.

[6] H. O. Anger, Scintillation camera with multichannel collimators, Journal of Nuclear Medicine 5 (7) (1964) 515-531.

[7] L. Mihailescu, K. P. Ziock, A. Raffo-Caiado, J. Hayward, S. Smith, J. Bogard, C. B. Boehnen, Combined measurements with three-dimensional design information verification system and gamma-ray imaging - a joint effort between oak ridge national laboratory, lawrence livermore national laboratory, and the joint research center at ispra, in: Proceedings of the 47th Annual INMM Meeting, INMM, 2006.

[8] L. Mihailescu, K. Vetter, D. Chivers, Standoff 3d gamma-ray imaging, Nuclear Science, IEEE Transactions on 56 (2) (2009) 479-486.

[9] S. Geman, D. E. McClure, Statistical methods for tomographic image reconstruction (1987).

[10] J. Hsieh, Computed tomography: principles, design, artifacts, and recent advances, SPIE Bellingham, WA, 2009.

[11] M. M. Ter-Pogossian, Positron emission tomography (pet), in: Diagnostic Imaging in Medicine, Springer, 1983, pp. 273-277.

[12] G. F. Knoll, Single-photon emission computed tomography, Proceedings of the IEEE 71 (3) (1983) 320-329.

[13] J. Tickner, M. Currie, G. Roach, Feasibility study for a low-cost 3d gammaray camera, Applied radiation and isotopes 61 (1) (2004) 67-71.

[14] Q. Looker, L. Stonehill, M. Wallace, M. Galassi, M. Cowee, E. Fenimore, W. V. McNeil, Demonstration of imaging via backscattering of annihilation gamma rays, Nuclear Instruments and Methods in Physics Research Section A: Accelerators, Spectrometers, Detectors and Associated Equipment 615 (3) (2010) 295-300.

[15] C. Domingo-Pardo, A new technique for 3d gamma-ray imaging: Conceptual study of a 3d camera, Nuclear Instruments and Methods in Physics Research Section A: Accelerators, Spectrometers, Detectors and Associated Equipment 675 (2012) 123-132. 
[16] K. Vetter, M. Burks, C. Cork, M. Cunningham, D. Chivers, E. Hull, T. Krings, H. Manini, L. Mihailescu, K. Nelson, et al., High-sensitivity compton imaging with position-sensitive si and ge detectors, Nuclear Instruments and Methods in Physics Research Section A: Accelerators, Spectrometers, Detectors and Associated Equipment 579 (1) (2007) 363-366.

[17] F. Endres, J. Hess, N. Engelhard, J. Sturm, D. Cremers, W. Burgard, An evaluation of the rgb-d slam system, in: Robotics and Automation (ICRA), 2012 IEEE International Conference on, IEEE, 2012, pp. 1691-1696.

[18] A. Haefner, D. Gunter, R. Barnowski, K. Vetter, A filtered back-projection algorithm for $4 \pi$ compton data, Transactions in Nuclear Science (under review).

[19] L. Parra, H. H. Barrett, List-mode likelihood: Em algorithm and image quality estimation demonstrated on 2-d pet, Medical Imaging, IEEE Transactions on 17 (2) (1998) 228-235. 